\title{
Duodenal angiomyolipoma with multiple systemic vascular malformations and aneurysms: A case report and literature review
}

\author{
YIQIU WANG ${ }^{1 *}$, XIULI ZHANG ${ }^{2 *}$, PENG LIU ${ }^{1}$, GUAN JIANG $^{2}$ and WENLOU LIU ${ }^{3}$ \\ ${ }^{1}$ Department of Surgical Oncology, Xuzhou Central Hospital, Southeast University Cancer Institute, Xuzhou, \\ Jiangsu 221009; ${ }^{2}$ Department of Radiology, Affiliated Hospital of Xuzhou Medical College, Xuzhou, Jiangsu 221006; \\ ${ }^{3}$ Department of Oncology, Zhongnan Hospital of Wuhan University, Hubei Key Laboratory of Tumor \\ Biological Behaviors and Hubei Cancer Clinical Study Center, Wuhan, Hubei 430071, P.R. China
}

Received July 10, 2016; Accepted July 7, 2017

DOI: $10.3892 / \mathrm{ol} .2017 .7011$

\begin{abstract}
Angiomyolipomas (AMLs) are barely benign mesenchymal tumors that usually occur in the kidneys and may be associated with tuberous sclerosis complex (TSC). Extrarenal AMLs are markedly rare and infrequently observed in the duodenum. In the present case report, a 22 -year-old female patient with duodenal AMLs presenting multiple systemic vascular malformations and aneurysms is described. The patient had a medical history of aneurysm rupture of the right subclavian artery and no other manifestation of TSC. Surgical intervention was performed. Following complete tumor resection, the patient declined to be treated further for vascular lesions. Pathological and immunohistochemical examination confirmed the diagnosis of duodenal AMLs. No tumor recurrence or progression of the vascular lesions was observed within 24 months of follow-up. This case report demonstrates the scarcity of duodenal AMLs with multiple systemic vascular malformations and aneurysms, which may be associated with novel gene mutations or TSC; however, further verification by gene sequencing is required.
\end{abstract}

\section{Introduction}

Angiomyolipomas (AMLs) are benign mixed mesenchymal neoplasms that arise predominantly in the kidneys (1).

Correspondence to: Dr Wenlou Liu, Department of Oncology, Zhongnan Hospital of Wuhan University, Hubei Key Laboratory of Tumor Biological Behaviors and Hubei Cancer Clinical Study Center, 169 Donghu Road, Wuhan, Hubei 430071, P.R. China

E-mail: liuwenlou@163.com

*Contributed equally

Abbreviations: AML, angiomyolipoma; BITA, bilateral internal thoracic artery; CT, computed tomography; CTA, CT angiogram; DSA, digital subtraction angiography; EDS, Ehlers-Danlos syndrome; SMA, superior mesenteric artery; TSC, tuberous sclerosis complex

Key words: angiomyolipoma, duodenum, vascular malformation, aneurysm, tuberous sclerosis
Extrarenal AMLs are rare and, in particular, AMLs of the duodenum are uncommon. To the best of our knowledge, only two cases have been reported based on a search of the PubMed and Medline databases (2,3). Among the extrarenal sites, the liver is the most frequently involved (4).

AMLs have been originally described as hamartomas or choristomas, formed of smooth muscle, abnormal blood vessels and fat tissue (5). However, evidence has indicated that AMLs belong to the family of perivascular epithelioid cell tumors (PEComas) (6). The first-line treatment of AMLs is surgical excision. Even though AMLs are considered to be benign tumors, they may be invasive and recurrent $(5,6)$.

The majority $(\sim 80 \%)$ of AMLs are sporadic, whereas the remainder are associated with tuberous sclerosis complex (TSC) (6,7). Most patients with TSC have renal manifestations, whereas extrarenal AMLs are usually not associated with TSC (8). The etiology is not completely understood. In the present case report, a rare AML case arising in the duodenum and presenting with multiple systemic vascular malformations and aneurysms is described.

\section{Case report}

A 22-year-old female patient, suffering from early satiety, intermittent upper abdominal pain and vomiting for 2 years, was admitted into the Zhongnan Hospital of Wuhan University (Hubei, China) in February 2013, with upper abdominal pain and symptoms of hematemesis. The patient had no previous history of TSC. Physical examination revealed moderate pallor and mild tenderness of the middle upper abdominal quadrant; however, the patient's vital signs were stable. No abnormalities in laboratory examinations, including blood biochemical tests, urinalysis and serum tumor markers; other than a low hemoglobin concentration $(82 \mathrm{~g} / \mathrm{l}$, compared with normal values of 120-160 g/l), were observed.

Esophagogastroduodenoscopy (upper gastrointestinal endoscopy) revealed multiple duodenal mucosa polypoid masses in the bowel wall of the descending duodenum (Fig. 1A). Biopsy from the growth indicated a benign lesion. However, it was not possible to perform an immunohistochemical examination, as the biopsy material was insufficient. Computed tomography (CT) of the abdomen revealed a 
$6.5 \times 3.3 \mathrm{~cm}$ well-circumscribed mass in the second part of the duodenum, formed predominantly of fat tissue and presenting a banded calcified center (Fig. 1B and C). Laparotomy was performed through an abdominal midline incision. During surgery, multiple mobile pedunculated polypoid masses with maximum diameter $3.5 \mathrm{~cm}$ were observed in the second part of the duodenum. These masses protruded into the duodenal lumen. Owing to the large volume of the lesion, the patient underwent duodenopancreatectomy and the tumor was completely resected.

Multiple well-circumscribed polypoid submucosal masses in the descending duodenum near the ampulla of Vater, with dimensions $6.5 \times 3.5 \times 2.2 \mathrm{~cm}$, were observed in gross pathological examination (Fig. 2A). Microscopically, they were primarily formed of mature adipose tissue, thick- or thin-walled blood vessels and interspersed areas of spindle-shaped smooth muscle fibers (Fig. 2B and C). Immunohistochemical staining was performed using an automated stainer (Benchmark, XT; Ventana Medical Systems, Inc., Tucson, AZ, USA) with 4- $\mu \mathrm{m}$ thick sections from formalin-fixed and paraffin-embedded tissues. Tissue sections were deparaffinized in xylene and rehydrated in a graded alcohol series. Following retrieval by the autoclave retrieval technique $(0.01 \mathrm{M}$ citrate buffer (pH 6.0); 10-20 min) and inhibition of endogenous peroxidase activity with $0.3 \% \mathrm{H}_{2} \mathrm{O}_{2}$ for $5-10 \mathrm{~min}$ at $37^{\circ} \mathrm{C}$, the slides were incubated with primary antibodies for $15-24 \mathrm{~min}$ at $37^{\circ} \mathrm{C}$. The primary antibodies used were $\alpha$-smooth muscle actin ( $\alpha$-SMA) monoclonal mouse (dilution, 1:200; catalog no., ab7817; Abcam, Cambridge, MA, USA), human melanoma black- 45 (HMB-45) monoclonal mouse (dilution, 1:100; catalog no., sc-59305; Santa Cruz Biotechnology, Inc., Dallas, TX, USA), and cluster of differentiation 34 (CD34) monoclonal mouse (dilution, 1:100; catalog no., sc-74499; Santa Cruz Biotechnology, Inc.). Negative controls were performed by omitting primary antibodies. After washing with Tris buffer, the slides were incubated for $30 \mathrm{~min}$ with a corresponding HRP Multimer polyclonal secondary antibody (ready-to-use; catalog no., G05739; Ventana Medical Systems, Inc.). The antigen-antibody reaction was visualized with 3,3'-diaminobenzidine (DAB) (Dako; Agilent Technologies $\mathrm{GmbH}$, Waldbronn, Germany). The sections were counterstained with hematoxylin for 2-3 min at room temperature, dehydrated and mounted. Results were interpreted using a light microscope at a magnification of $x 200$. Immunohistochemically, the proliferative smooth muscle tissue was positive for $\alpha$-smooth muscle actin ( $\alpha$-SMA) (Fig. 2D). Focal positivity with HMB-45 was also observed (Fig. 2E). The vascular components were immunoreactive for the hematopoietic progenitor cell antigen cluster of differentiation 34 (CD34) (Fig. 2F). The pathological diagnosis was AML.

No significant postoperative complications were observed. However, on the eighth postoperative day, hematemesis recurred $(\sim 60 \mathrm{ml})$. Further inquiry revealed a history of aneurysm rupture of the right subclavian artery. An emergency digital subtraction angiography (DSA) did not detect any evident bleeding point in the gastrointestinal tract. However, a narrow segment of the superior mesenteric artery with beaded appearance, relatively narrow distal vascular wall and possible aneurysmal dilatation was observed (Fig. 3A). In addition, marked dilatation and tortuosity of the inferior mesenteric artery was observed (Fig. 3B). Furthermore, severe tortuosity and variation of the right intercostal artery were also observed (Fig. 3C).

Subsequent CT angiography (CTA) and three-dimensional analyses further confirmed the existence of multiple systemic vascular alterations and aneurysms. Additionally, severe tortuosity and malformations of the right intercostal and the bilateral internal thoracic arteries, responsible for the blood supply to the liver and the spleen in the absence of the celiac trunk, were observed (Fig. 4).

Following treatment, the patient was stable and discharged from the hospital 20 days after surgery. Follow-up visits to the interventional center of the Zhongnan Hospital of Wuhan University for further evaluation and treatment were recommended. Endovascular aneurysm repair was suggested as a treatment strategy for the abdominal vascular aneurysms. However, the patient did not elect to have treatment. The patient was in good health and no signs of recurrence or progression of the patient's vascular lesions were detected in the first 24 follow-up months. Written informed consent was obtained from the patient prior to publication of the present study.

\section{Discussion}

AMLs are histologically defined as benign mesenchymal hamartomas that contain at least two mesenchymal tissue components. They were first described in the kidneys in 1951 by Morgan et al (7). The vast majority of AMLs primarily involve one or both kidneys. However, extrarenal AMLs have also been reported in several tissues, including the mediastinum, the retroperitoneum, the abdominal wall, the heart, the lungs, the stomach, the spleen, the bones, the transverse colon, the vagina, the upper lip, the nasal cavity, the spinal cord, the parotid glands and the skin (5,9-13). Duodenal AMLs are markedly rare and present clinically with satiety, melena, anemia, generalized weakness, vomiting and abdominal pain $(2,3)$.

Endoscopic diagnosis of extrarenal AMLs is challenging. Usually, CT and magnetic resonance imaging are sufficient to diagnose AMLs which are depicted as fat-containing lesions with abnormal vascularity (14). Radiological diagnosis and endoscopic ultrasound are the methods of choice for the evaluation of these tumors, but they do not have diagnostic value. Surgical excision is the first-line treatment for tumors with diameter larger than $4 \mathrm{~cm}$. However, inadequate resection may result in rapid local relapse (15). Minja et al (16) reported that selective arterial embolization has been used effectively to control the hemorrhagic lesions of patients with extrarenal AMLs.

Histologically, the classic AML contains various proportions of vascular tissue, smooth muscle and adipose tissue (17). However, in certain cases, one of the components may predominate or be virtually absent. AMLs with a large component of epithelioid cells resemble PEComas, a group of tumors originating from perivascular epithelioid cells (6). Even though AMLs are considered to be benign lesions, malignant behavior has been observed in epithelioid angiomyolipomas (18). However, malignant features in AMLs arising in the gastrointestinal tract have not been reported to 

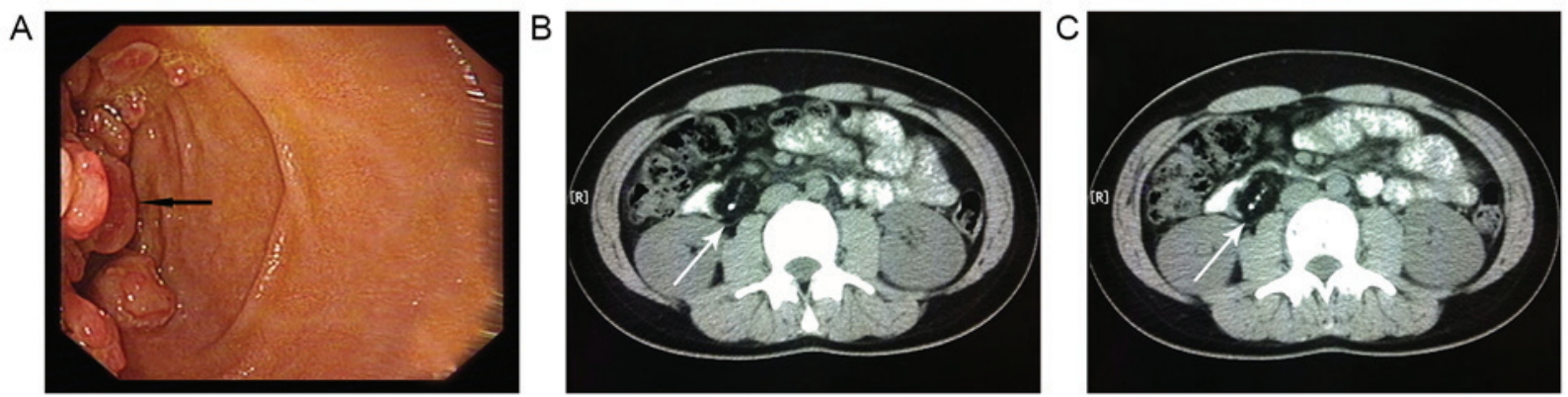

Figure 1. Esophagogastroduodenoscopy imaging and abdominal CT scan results. (A) Upper gastrointestinal endoscopy revealed multiple duodenal mucosa polypoid masses (black arrow) in the bowel wall of the descending duodenum. (B) A fat-density lesion, sized $6.5 \times 3.3 \mathrm{~cm}$ and (C) a banded calcification in the center of the second part of the duodenum (white arrow) were observed on sequential axial CT imaging of the upper abdomen. CT, computed tomography; R, right.
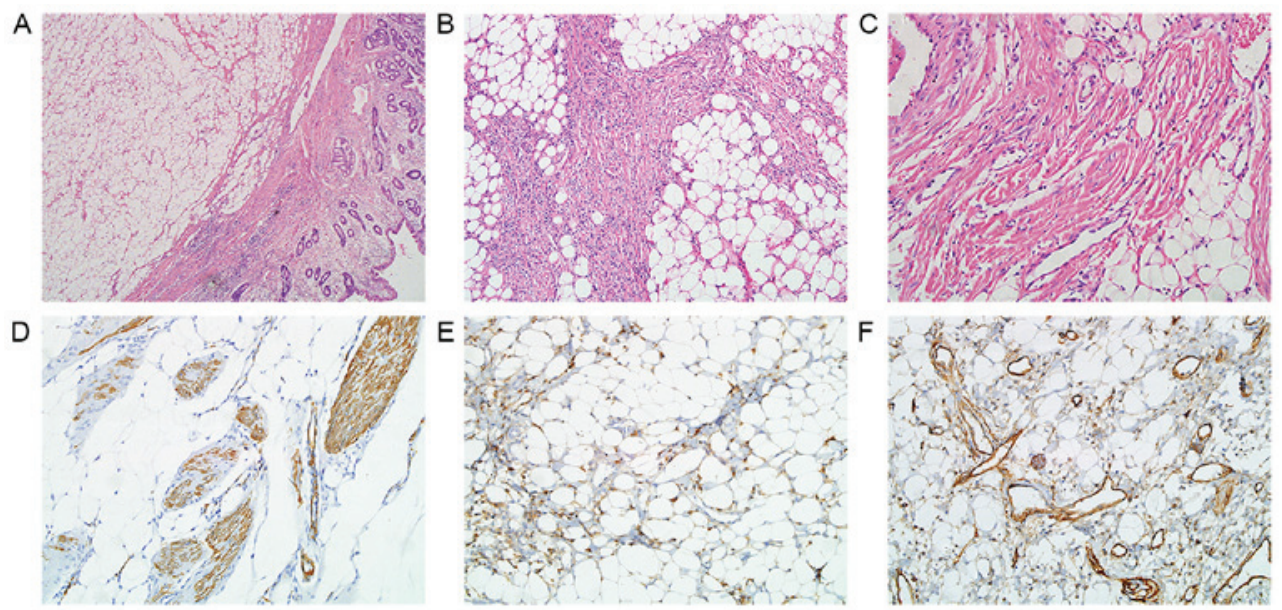

Figure 2. Pathological examination of the polypoid submucosal masses in the descending duodenum. (A) Histopathological examination revealed well-circumscribed lesions with overlying duodenal mucosa (H\&E staining; magnification, $\mathrm{x} 40$ ). Mature adipose tissue with thick- or thin-walled blood vessels and proliferative spindle-shaped smooth muscle fibers [H\&E staining; magnification, (B) x100 and, (C) x200]. Immunohistochemical examination demonstrated positivity of (D) $\alpha$-smooth muscle actin (E), human melanoma black-45 and (F) the hematopoietic progenitor cell antigen cluster of differentiation 34 (magnification, x200). H\&E, hematoxylin and eosin.
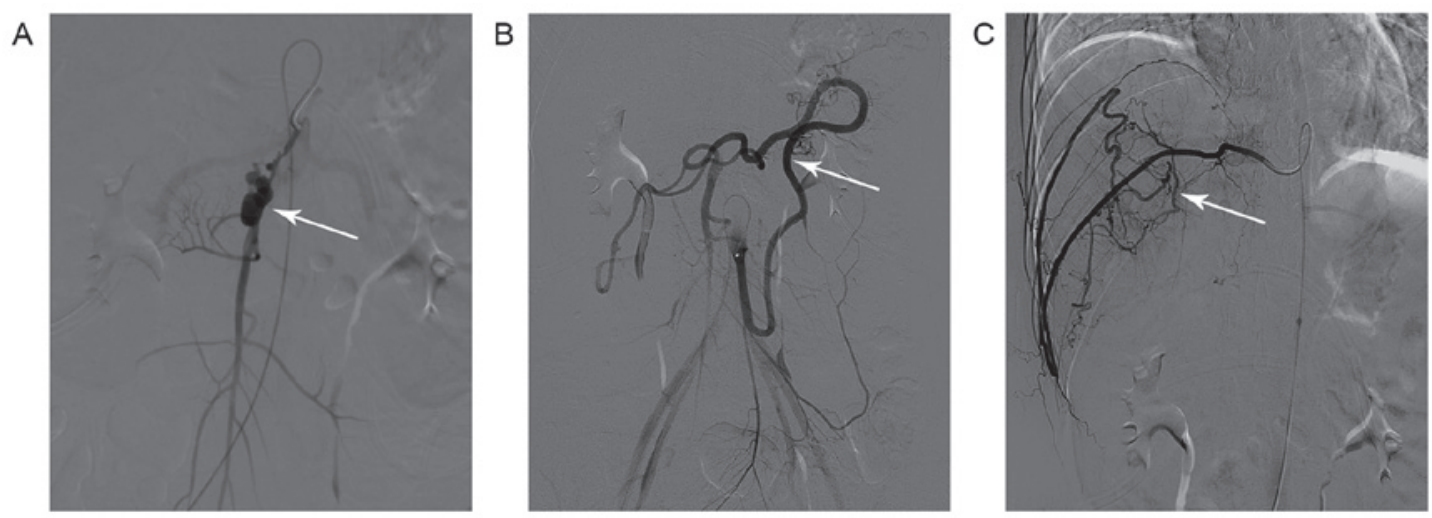

Figure 3. Digital subtraction angiography revealed (A) stenosis and aneurysmal dilatation (white arrow) of the initial segment of the superior mesenteric artery, (B) evident dilatation and tortuosity (white arrow) of the inferior mesenteric artery, and (C) marked tortuosity and alterations (white arrow) of the right intercostal artery.

date. Positive immunoreactivity for $\alpha$-SMA and HMB-45 is typical of AMLs and may be used to distinguish AMLs from other similar lesions including angiolipomas, angioleiomyomas, liposarcomas and leiomyosarcomas $(13,15)$. However, certain cases of gastrointestinal AML demonstrated weak or no immunoreactivity for HMB-45 in spindle cells $(3,15)$. The patient described in the present case report was positive for $\alpha$-SMA and only focal positive for HMB45, whereas the 


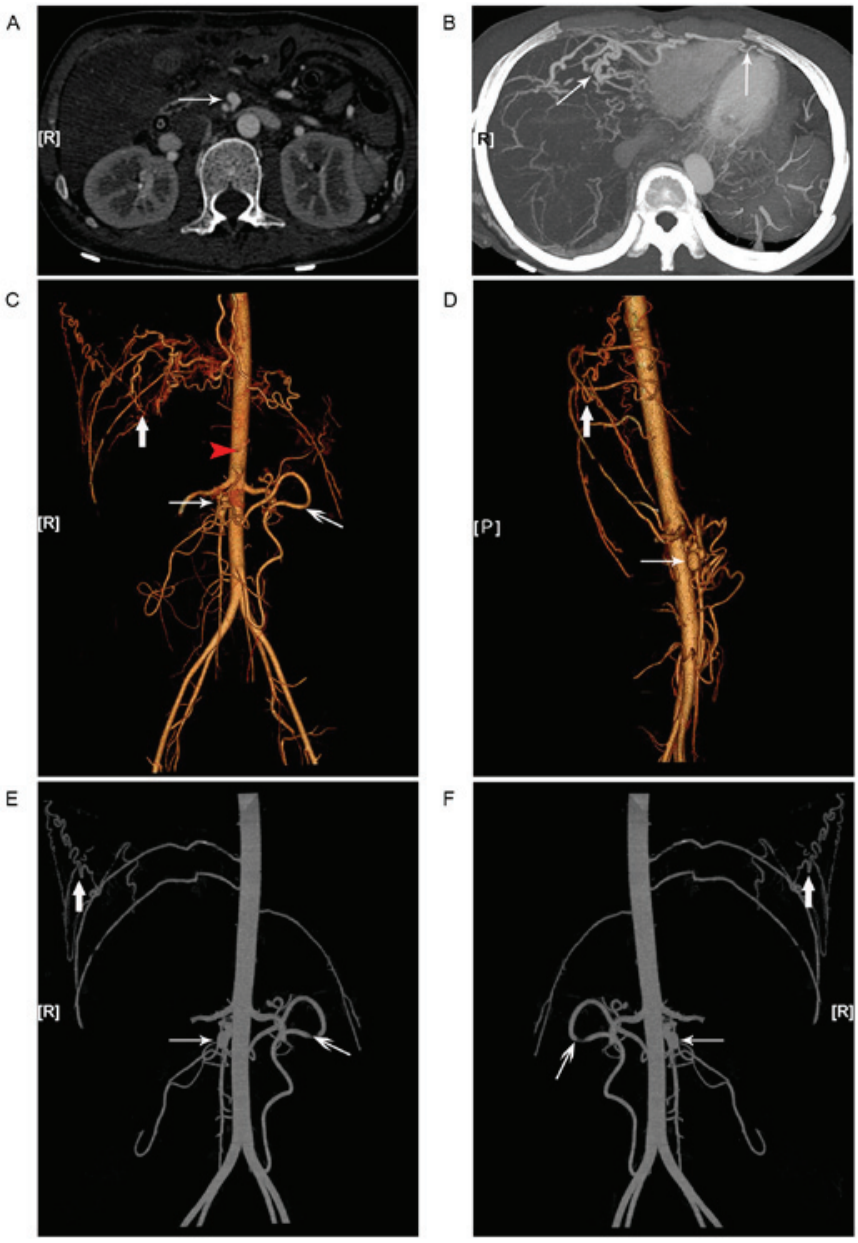

Figure 4. CTA and three-dimensional analyses demonstrated the existence of multiple systemic vascular malformations and aneurysms. (A) Postoperative CTA revealed aneurysmal dilatation (white arrow) of the SMA and (B) severe tortuosity and alterations of the BITA (white arrow) in axial view. $(\mathrm{C}$ and $\mathrm{D})$ Volume rendering and maximum intensity projection ( $\mathrm{E}$ and F) CT images demonstrating the absence of the celiac trunk (red arrow), a severely narrow and tortuous initial segment of the SMA presenting aneurysmal dilatation (thin arrow), marked dilatation and tortuosity of the inferior mesenteric artery (middle arrow) and remarkable tortuosity and alterations of the right intercostal artery and the BITA (thick arrow). BITA, bilatera internal thoracic artery; CTA, computed tomography angiography; SMA, superior mesenteric artery; $\mathrm{P}$, posterior; $\mathrm{R}$, right.

vascular component was positive for CD34. These results are consistent with those of previously reported cases of duodenal AML (2,3).

Toye and Czarnecki (2) reported the first case of duodenal AML in a 60-year-old female presenting with anemia, satiety and a 36x36 mm well-circumscribed duodenal mass. De Padua et al (3) reported another case in a 66-year-old male presenting with generalized weakness, severe anemia (hemoglobin concentration $6 \mathrm{mg} / \mathrm{dl}$ ), melena and a 40x40 mm pedunculated polyp duodenal mass. The two lesions demonstrated a single polypoid pattern, whereas in the patient described in the present case report, multiple polypoid neoplasms were observed. None of the three cases was associated with tuberous sclerosis. The three patients underwent surgical excision and no recurrence was observed.

The majority of AMLs are sporadic (80\%) and a total of $20 \%$ are associated with TSC or lymphangioleiomyomatosis $(7,18)$. TSC, a hereditary syndrome with autosomal dominant inheritance, is associated with benign tumors in a number of organs, including the skin, the brain, the kidneys, the heart and the lungs (19). AMLs occur in between 55 and $80 \%$ of patients and are the most common cause of TSC-associated mortality in adults $(8,19)$. Abnormal vasculature is a typical feature of AMLs. Aneurysmal dilatations of intratumoral vessels and micro- or macro-aneurysms, associated with an increased risk of hemorrhage, are frequently observed $(19,20)$. It is worthy of mention that patients with Ehlers-Danlos syndrome (EDS) type IV display similar severe arterial complications, which cannot be differentially diagnosed. However, the diagnosis of type IV EDS is confirmed by a mutation in the type III procollagen gene (21).

Certain limitations to the treatment protocol followed in the present case report are discussed below. First, detailed medical history of this patient was not initially obtained. Further inquiry revealed a history of aneurysm rupture in the right subclavian artery. Thus, a preoperative digital angiogram on the duodenal AML was not performed. Secondly, due to a subjective diagnosis of benign polyps, no postoperative macroscopic sample was obtained. The postoperative DSA and CTA revealed multiple systemic vascular malformations and aneurysms. However, there is no solid evidence that these multiple vascular lesions are certainly associated with TSC. Currently, the diagnosis of TSC is based on clinical observations. Molecular genetic sequencing is not recommended as a diagnostic tool for TSC, due to the complexity and variability of genetic mutations (22). In the patient described in the present case report, no evidence of other lesions associated with TSC was obtained. Examination of genetic mutations is required to confirm the final diagnosis. However, the patient was unwilling to accept it.

Duodenal AMLs are markedly rare benign tumors. Their differential diagnosis is complicated and challenging, particularly in cases arising in the duodenum, presenting with multiple vascular lesions. Surgical excision is considered the most effective treatment. Even though molecular genetic sequencing was not performed, the available data suggest that this case may not be associated with TSC. However, further research is required to elucidate the genetic mutations associated with AML presenting with multiple systemic vascular malformations and aneurysms.

\section{Acknowledgements}

The authors would like to thank Dr Chao Qin (Department of Urology, The First Affiliated Hospital of Nanjing Medical University, Jiangsu, China) for his assistance with the language editing of the present paper before submission.

\section{References}

1. Tong YC, Chieng PU, Tsai TC and Lin SN: Renal angiomyolipoma: Report of 24 cases. Br J Urol 66: 585-589, 1990.

2. Toye LR and Czarnecki LA: CT of a duodenal angiomyolipoma [corrected]. AJR Am J Roentgenol 178: 92, 2002.

3. De Padua M, Gupta N, Broor SL and Govil D: Duodenal angiomyolipoma: A case report. Indian J Pathol Microbiol 50: 568-569, 2007.

4. Petrolla AA and Xin W: Hepatic angiomyolipoma. Arch Pathol Lab Med 132: 1679-1682, 2008.

5. Candas F, Berber U, Yildizhan A, Yiyit N, Görür R and Isitmangil T: Anterior mediastinal angiomyolipoma. Ann Thorac Surg 95: 1431-1432, 2013. 
6. Lienert AR and Nicol D: Renal angiomyolipoma. BJU Int 110 (Suppl 4): S25-S27, 2012.

7. Morgan GS, Straumfjord JV and Hall EJ: Angiomyolipoma of the kidney. J Urol 65: 525-527, 1951.

8. Curatolo P, Bombardieri R and Jozwiak S: Tuberous sclerosis. Lancet 372: 657-668, 2008

9. Ramírez Daniel L, García Sabela L, Rey Jorge R and Calvo Antonio O: Retroperitoneal angiomyolipoma: Review of literature and report of a new case. Actas Urol Esp 34: 815-817, 2010 (In Spanish).

10. Abdulkader M, Abercrombie J, McCulloch TA and Kaye PV: Colonic angiomyolipoma with monotypic expression and a predominant epitheloid component. J Clin Pathol 58: 1107-1109, 2005.

11. Helwig K, Talabiska D, Cera P and Komar M: Gastric angiomyolipoma: A previously undescribed cause of upper GI hemorrhage. Am J Gastroenterol 93: 1004-1005, 1998.

12. Rosado P, Villalain L, De Vicente JC, Vivanco B and Torre A: Angiomyolipoma of the parotid gland: Report of a case and review of the literature. J Oral Maxillofac Surg 68: 2609-2612, 2010.

13. da Silva AA, Carlos R, Contreras E, de Almeida OP, Lopes MA and Vargas PA: Angiomyolipoma of the upper lip: Case report and review of the literature. Med Oral Patol Oral Cir Bucal 12: E101-E104, 2007.

14. Israel GM, Bosniak MA, Slywotzky CM and Rosen RJ: CT differentiation of large exophytic renal angiomyolipomas and perirenal liposarcomas. AJR Am J Roentgenol 179: 769-773, 2002.

15. Lee CH, Kim JH, Yang DH, Hwang Y, Kang MJ, Kim YK and Lee MR: Ileal angiomyolipoma manifested by small intestinal intussusception. World J Gastroenterol 15: 1398-1400, 2009.
16. Minja EJ, Pellerin M, Saviano $\mathrm{N}$ and Chamberlain RS: Retroperitoneal extrarenal angiomyolipomas: An evidence-based approach to a rare clinical entity. Case Rep Nephrol 2012: 374107, 2012.

17. Boorjian SA, Frank I, Inman B, Lohse CM, Cheville JC, Leibovich BC and Blute ML: The role of partial nephrectomy for the management of sporadic renal angiomyolipoma. Urology 70: 1064-1068, 2007.

18. Hassan M, El-Hefnawy AS, Elshal AM, Mosbah A, El-Baz M and Shaaban A: Renal epithelioid angiomyolipoma: A rare variant with unusual behavior. Int Urol Nephrol 46: 317-322, 2014.

19. De Waele L, Lagae L and Mekahli D: Tuberous sclerosis complex: The past and the future. Pediatr Nephrol 30: 1771-1780, 2015.

20. Guridi J, Tuñón M, Caballero C, Gallo-Ruiz A, Vázquez A and Zazpe I: Intracranial hemorrhage from an arteriovenous malformation (AVM) in a tuberous sclerosis patient. Neurologia 16: 281-284, 2001 (In Spanish).

21. Germain DP: Ehlers-Danlos syndrome type IV. Orphanet J Rare Dis 2: 32, 2007.

22. Sancak O, Nellist M, Goedbloed M, Elfferich P, Wouters C, Maat-Kievit A, Zonnenberg B, Verhoef S, Halley D and van den Ouweland A: Mutational analysis of the TSC1 and TSC2 genes in a diagnostic setting: Genotype-phenotype correlations and comparison of diagnostic DNA techniques in Tuberous Sclerosis Complex. Eur J Hum Genet 13: 731-741, 2005. 